\title{
Multi-Plet Two-Channel Perfect Reconstruction Filter Banks
}

\author{
S. C. Chan and K. M. Tsui
}

Department of Electrical and Electronic Engineering, The University of Hong Kong, Pokfulam Road, Hong Kong.

\begin{abstract}
This paper proposes a new class of two-channel structural perfect reconstruction (PR) FIR filter banks (FBs) called the multi-plet FB. It generalizes structural PR FBs proposed by Phoong et al. and triplet FBs by employing multiple lifting steps similar to the conventional lifting structure. Apart from the important structural PR property, the multi-plet FB can be systematically designed to meet a given specification on the passband/stopband ripples and transition bandwidth. A low order prototype $\mathrm{PR}$ FB with a much wider transition band is first designed in order to obtain prescribed passband and stopband ripples. A subfilter is then designed so that the prototype FB can be wrapped by means of frequency transformation to meet the desired transition bandwidth, while preserving the PR condition, passband/stopband ripples and lifting structure. The design procedure is very general and it can be applied to both linearphase and low-delay multi-plet FBs. Design examples show that the proposed approach is more flexible in controlling the frequency characteristics of the PR FBs and has a lower design complexity than conventional methods.
\end{abstract}

\section{INTRODUCTION}

PR FBs have important applications in signal analysis, coding and the design of wavelet bases. An efficient structure of two-channel FIR/IIR FBs, which structurally satisfies the PR condition, is the structural PR FB proposed by Phoong et al. [1]. One limitation of this structure is that the magnitudes of the lowpass and highpass analysis filters at $\omega=\pi / 2$ in the linearphase case are respectively restricted to 0.5 and 1 , or vice versa. In another structural PR FBs called triplet FBs [2], a generalization of the structure in [1], more symmetric frequency responses can be obtained by properly choosing the parameters in the structure. The triplet FBs are parameterized by three subfilters and three delay parameters. To meet different design specifications, these subfilters can be chosen as arbitrary functions such as linear-phase FIR [2], [4] and low-delay FIR [5] functions. Because of this property, the design of PR FBs can be simplified to general filter design problems.

The flexibility and effectiveness of these structural PR FBs motivate us to study further a new class of two-channel structural PR FBs with multiple lifting steps called the multi-plet FBs. It extends the structural PR FBs [1] and the triplet FBs [2], which involve two and three lifting steps, respectively. It is shown later in this paper that the design of the proposed multi-plet FB is closely related to the concept of frequency transformation of digital filters studied in [6] and [7]. As a result, the frequency characteristics of the multi-plet PR FB can be varied by varying on-line the subfilter as variable digital filter [8]. More precisely, if a two-channel PR FB is expressed in certain ladder or lifting structure [3] having the same subfilter of the form $\left(1+z^{-1}\right) / 2$ in all the lifting steps, then appropriate frequency transformation, similar to the approaches in [6] and [7], can be applied directly to the lifting structure to obtain another PR FB with the same number of lifting steps, the same passband and stopband ripples, but an arbitrary sharp transition bandwidth. Thus, the design of the multi-plet FB can be divided into two sub-problems: (i) the design of a prototype PR FB to meet certain specifications on the passband and stopband ripples and (ii) the design of a subfilter which determines the cutoff frequency of the final multi-plet FB after transformation. Like the structural PR and triplet FBs, the $\mathrm{PR}$ condition is preserved under coefficient quantization.
Another important advantage is that a systematic design procedure and design formulae are available to meet a given specification on the passband and stopband ripples and transition bandwidth of the final PR FB. The proposed design approach is also applicable to the design of low-delay multi-plet FBs, where low-delay FIR subfilters are used instead of linear-phase FIR subfilters for reducing the system delay. This gives rise to a new class of low-delay multiplet PR FB, which is a generalization of those in [9] and [5] with two and three lifting steps, respectively. Design results show that the proposed low-delay multi-plet FB offers a comparable performance as, but considerably lower system delay than, their linear-phase counterparts. The design of the linear-phase and low-delay subfilters can be solved using second order cone programming (SOCP) [10]. The main advantage of using SOCP is that the problem is convex and the global optimal solution, if it exists, is guaranteed. Furthermore, using the SOCP formulation, it is possible to impose prescribed $K$-regularity constraints on the multi-plet FBs in order to obtain muti-plet-based wavelet bases. The required $K$-regularity condition is a set of linear equality constraints on the subfilter. Due to page limitation, the problem of imposing $K$-regularity condition will be reported elsewhere.

It should be noted that there was previous work on the design of two-channel PR FBs using frequency transformation [11]. The present work differs from [11] in that the transformation is applied directly to the lifting structure, which leads naturally to efficient implementation, and the PR condition is unaffected by coefficient quantization. In addition, thanks to the frequency transformation concept in [6], [7], it is possible to derive systematic procedure and design formulae to meet the given specifications of passband and stopband ripples and transition bandwidth. On the other hand, these relations between the prototype $\mathrm{FB}$ and the transformed FB were not explored in [11] so that the frequency characteristics of the latter cannot be precisely controlled. Finally, only linear-phase PR FBs, but not low-delay PR FBs, were studied in [11]. The paper is organized as follows: the proposed multi-plet FBs and the concept of frequency transformation are introduced in section II. Several design examples are given in section III, and finally, conclusion is drawn in section IV.

\section{Multi-Plet Two-Channel PR FIR FBs}

\section{A. - Lifting Structure}

The general structure of the multi-plet two-channel FBs is shown in figure 1. It is parameterized by $L$ subfilters $Q_{l}(z), L$ delay parameters $N_{l}, L$ lifting coefficients $p_{l}$, and two scaling constants $C_{0}$ and $C_{1}$ for $l=0,1, \ldots, L-1$. It can be seen from figure 1 that the $\mathrm{z}$-transform of the intermediate filter $H^{(l)}(z)$ after the $l$-th lifting step can be written as the following recursion:

$$
\begin{aligned}
& H^{(0)}(z)=z^{-2 N_{0}-1}+p_{0} \cdot Q_{0}\left(z^{2}\right), \\
& H^{(1)}(z)=z^{-2 N_{1}}+p_{1} \cdot Q_{1}\left(z^{2}\right) H^{(0)}(z), \\
& H^{(l)}(z)=z^{-2 N_{l}} H^{(l-2)}(z)+p_{l} \cdot Q_{l}\left(z^{2}\right) H^{(l-1)}(z), \\
& \text { for } l=2,3, \ldots, L-1 .
\end{aligned}
$$

Hence, the z-transforms of the resultant analysis and synthesis filters in the lifting structure can be written as follows:

$$
\begin{aligned}
& H_{0}(z)=C_{0} H^{(L-2)}(z), H_{1}(z)=C_{1} H^{(L-1)}(z), \\
& F_{0}(z)=H_{1}(-z) \text { and } F_{1}(z)=-H_{0}(-z) .
\end{aligned}
$$


We shall consider a special case of lifting with identical subfilters:

$$
Q_{L-1}\left(z^{2}\right)=Q_{L-2}\left(z^{2}\right)=\cdots=Q_{1}\left(z^{2}\right)=Q_{0}\left(z^{2}\right)=Q\left(z^{2}\right),
$$

The delay parameters $N_{l}$ are then given by:

$$
N_{L-1}=\cdots=N_{2}=N_{1}=G \text { and } N_{0}=(G-1) / 2,
$$

where $G$ is the passband group delay of $Q\left(z^{2}\right)$. As a result, the group delays of the analysis filter pair, $H_{0}(z)$ and $H_{1}(z)$, are respectively given by:

$$
G_{0}=(L-1) \cdot G \text { and } G_{1}=L \cdot G .
$$

\section{B. - Transformation of Lifting Structure}

If the lifting coefficients are properly chosen, the multi-plet FB is structurally PR with desirable frequency characteristics for arbitrary choice of the subfilters which can be chosen as linearphase/low-delay FIR or IIR filters. However, the determination of these coefficients is a non-trivial task since the lifting factorization using Euclidean factorization [3] is in general nonunique and there are many possible choices of these coefficients. To overcome this problem, a prototype PR FB with type 1 FIR linear-phase analysis filters (i.e. filters with odd length and symmetric impulse response) is employed so that it can be factored uniquely into a ladder or lifting structure having the same subfilter of the form $Q(z)=\widetilde{Q}(z)=\left(1+z^{-1}\right) / 2$ in all the lifting steps [12]. It then follows that the zero-phase responses of $\widetilde{H}^{(l)}(z)$, denoted by $\widetilde{R}^{(l)}(x)$, for $l=0,1, \ldots, L-1$ are given by:

$$
\begin{aligned}
& \widetilde{R}^{(0)}(x)=z \cdot \widetilde{H}^{(0)}(z)=1+p_{0} x, \\
& \widetilde{R}^{(1)}(x)=z^{2} \cdot \widetilde{H}^{(1)}(z)=1+p_{1} x \cdot \widetilde{R}^{(0)}(x), \\
& \widetilde{R}^{(l)}(x)=z^{l+1} \cdot \widetilde{H}^{(l)}(z)=\widetilde{R}^{(l-2)}(z)+p_{l} x \cdot \widetilde{R}^{(l-1)}(x),
\end{aligned}
$$$$
\text { for } l=2,3, \ldots, L-1 \text {. }
$$

where $x=z \cdot \widetilde{Q}\left(z^{2}\right)=\left(z+z^{-1}\right) / 2$. In general, the zero-phase responses of the analysis filters can be expressed as follows:

$$
\widetilde{R}_{0}(x)=C_{0}\left[1+\sum_{n=1}^{L-1} a_{0}(n) x^{n}\right] \text { and } \widetilde{R}_{1}(x)=C_{1}\left[1+\sum_{n=1}^{L} a_{1}(n) x^{n}\right] .
$$

For convenience, the corresponding causal FB considered in (7) is referred to as the prototype FB. We can see that the lengths of the analysis filters are odd, and differed by two. With the following substitution of variable:

$$
x=R_{Q}(\hat{x})=z^{G} Q\left(z^{2}\right),
$$

where $R_{Q}(\hat{x})$ is the zero-phase response of the subfilter $Q\left(z^{2}\right)$ with certain integer $G$, we obtain from (7) the zero-phase response of a new analysis filter pair as follows:

$$
\begin{aligned}
& R_{0}(\hat{x})=C_{0}\left\{1+\sum_{n=1}^{L-1} a_{0}(n)\left[R_{Q}(\hat{x})\right]^{n}\right\} \text { and } \\
& R_{1}(\hat{x})=C_{1}\left\{1+\sum_{n=1}^{L} a_{1}(n)\left[R_{Q}(\hat{x})\right]^{n}\right\} .
\end{aligned}
$$

We call the corresponding casual FB the transformed FB of the prototype. Since the transformed FB is obtained by replacing $x$ in each lifting step of figure 1 by $R_{Q}(\hat{x})$, it can also be implemented by the same number of lifting steps as the prototype.

To analyze the effect of the transformation, let us express the $z$-variable as $e^{j \widetilde{\omega}}$, where $\widetilde{\omega}$ is the digital radian frequency of the prototype FB. From (8), the digital frequencies before and after transformation are related by:

$$
x=\cos (\widetilde{\omega})=R_{Q}(\hat{x})=R_{Q}(\cos (\omega)),
$$

where $\hat{x}=\cos \omega$ and $\omega$ is the digital radian frequency of the transformed FB. Figure 2 shows graphically the relationship of the various quantities in the transformation. It can be seen that $x$ varies monotonically from -1 to 1 as $\widetilde{\omega}$ varies from $-\pi$ to $\pi$, and the transition band of the prototype FB lies in the interval around $\widetilde{\omega}=\pi / 2$ or $x=0$. Therefore, if $R_{Q}(\hat{x})$ is appropriately designed to have a sharper characteristics than $\hat{x}=\cos \omega$ around $\omega=\pi / 2$, then the transformed FB will have a much narrower transition band. Furthermore, if we want to preserve the passband and stopband ripples of the prototype FB in the transformed $\mathrm{FB}, R_{Q}(\hat{x})$ should map respectively the values of $x=\cos \widetilde{\omega}$ in the passband and stopband of the prototype FB to the new passband and stopband of the transformed FB.

Using these results, the design of a two-channel PR FB can be splitted into two much simpler sub-problems, namely the designs of the prototype PR FB and the subfilter $R_{O}(\hat{x})$. The advantage of this approach is that the length of the prototype FB, which is targeted to have rather wide transition bandwidth, are relatively short. Therefore, the number of variables, and hence the design complexity of the prototype FB can be greatly reduced, as compared to the direct design of the target FB using nonlinear constrained optimization with large number of variables in order to satisfy the specifications. On the other hand, the transformed FB can achieve a much narrower and prescribed transition bandwidth by properly designing the subfilter using conventional filter design technique, while preserving the passband/stopband ripples of the prototype FB. Next, we shall determine the specifications of the prototype FB and the subfilters.

\section{C. - Design Specifications and methodology}

Obviously, the target specifications are identical to those of the transformed FB. Suppose that the specifications of the latter are as follows:

$$
\begin{aligned}
& \text { Lowpass: } \begin{cases}1-\delta_{p 0} \leq R_{0}(\hat{x}) \leq 1+\delta_{p 0}, & 0 \leq \omega \leq \omega_{c} \\
\delta_{s 0} \leq R_{0}(\hat{x}) \leq \delta_{s 0}, & \pi-\omega_{c} \leq \omega \leq \pi\end{cases} \\
& \text { Highpass: }\left\{\begin{array}{ll}
1-\delta_{p 1} \leq R_{1}(\hat{x}) \leq 1+\delta_{p 1}, & \pi-\omega_{c} \leq \omega \leq \pi \\
\delta_{s 1} \leq R_{1}(\hat{x}) \leq \delta_{s 1}, & 0 \leq \omega \leq \omega_{c}
\end{array},\right.
\end{aligned}
$$

where $\omega_{c}$ specifies the cutoff frequency; $\delta_{p i}$ and $\delta_{s i}$ are respectively the passband and stopband ripples of the analysis filters $H_{i}(z)$ and hence $R_{i}(\hat{x})$, for $i=0,1$. As mentioned earlier, the target specification can be met by first designing a prototype $\mathrm{FB}$ with the given passband and stopband ripples but with a wider transition band. After appropriate frequency transformation in (8), the given transition bandwidth can be achieved, while preserving the lifting structure, PR property, and passband/stopband ripples. Therefore, the specifications of the prototype FB can be expressed as follows:

$$
\begin{aligned}
& \text { Lowpass: } \begin{cases}1-\delta_{p 0} \leq \widetilde{R}_{0}(x) \leq 1+\delta_{p 0}, & 0 \leq \widetilde{\omega} \leq \widetilde{\omega}_{c} \\
\delta_{s 0} \leq \widetilde{R}_{0}(x) \leq \delta_{s 0}, & \pi-\widetilde{\omega}_{c} \leq \widetilde{\omega} \leq \pi\end{cases} \\
& \text { Highpass: } \begin{cases}1-\delta_{p 1} \leq \widetilde{R}_{1}(x) \leq 1+\delta_{p 1}, & \pi-\widetilde{\omega}_{c} \leq \widetilde{\omega} \leq \pi \\
\delta_{s 1} \leq \widetilde{R}_{1}(x) \leq \delta_{s 1}, & 0 \leq \widetilde{\omega}_{c} \leq \widetilde{\omega}_{1}\end{cases}
\end{aligned}
$$

where $\widetilde{\omega}_{c}$ is the cutoff frequency of the prototype FB. On the other hand, it can be seen from Figure 2 that to transform the ripples of the prototype FB at the $\widetilde{\omega}$-domain to the frequency of interest at the $\omega$-domain, the subfilter should satisfy:

$$
\left\{\begin{array}{ll}
\cos \left(\widetilde{\omega}_{c}\right) \leq R_{Q}(\hat{x}) \leq 1, & 0 \leq \omega \leq \omega_{c} \\
-1 \leq R_{Q}(\hat{x}) \leq-\cos \left(\widetilde{\omega}_{c}\right), & \pi-\omega_{c} \leq \omega \leq \pi
\end{array} .\right.
$$

If the prototype FB is monotonic decreasing at the stopband, say a maximally-flat function in [11], then by choosing an appropriate subfilter, an arbitrary small stopband attenuation can be achieved after transformation. The basic idea is similar to the design procedures of the structural PR FB in $[1,8]$ and the triplet FB in $[2,4,5]$, except that the prototype FBs in these FBs are fixed and have simple coefficients. In addition, since the design of these FBs is not viewed as a transformation, each subfilter is usually designed independently. Alternatively, a set of prototype 
PR FBs with different passband/ stopband ripples can be designed offline. The subfilter can then be designed so that the transformed FB will be able to achieve a narrower transition bandwidth, while preserving the ripples of the prototype FB. Optimized prototype FBs usually lead to a better performance.

\section{D. - Design of Subfilters}

Although our discussion so far focuses on linear-phase FIR subfilters, the proposed transformation method also works well for approximately linear-phase FIR subfilters. The advantage of using low-delay subfilters over their linear-phase counterparts is that the overall system delay of the transformed FBs can be reduced. To begin with, denote the transfer function of the FIR subfilter to be designed by:

$$
Q(z)=\sum_{n=0}^{L_{Q}-1} q(n) z^{-n},
$$

where $L_{Q}$ and $q(n)$ are respectively the length and the impulse response of the subfilter. By introducing a delay term $e^{-j \omega G}$ into (12), the desired response of the subfilter can be rewritten as follows:

$$
Q_{d}(\omega)=m \cdot e^{-j \omega G / 2}, 0 \leq \omega \leq 2 \omega_{c},
$$

where $m=\left[1+\cos \left(\widetilde{\omega}_{c}\right)\right] / 2$. From (13) and (14), we also have:

$$
G=L_{Q}-1-D,
$$

where $D$ is a prescribed delay parameter. When $D=0$, the subfilter is an even-length linear-phase FIR filter with symmetric impulse response. Consequently, $G$ has to be an odd positive integer. Moreover, it was found that the subfilter length $L_{Q}$ is approximately given by:

$$
L_{Q} \approx \frac{-20 \log _{10}\left(\delta_{Q}\right)-5.1749}{4.5404 \cdot\left(\pi-2 \omega_{c}\right)},
$$

where $\delta_{Q}=\left[1-\cos \left(\widetilde{\omega}_{c}\right)\right] / 2$. This provides a good starting point to determine $L_{Q}$ to meet a given specification as shown in (12). For the low-delay case, $L_{Q}$ can be chosen as either odd or even integer, provided that $G$ is an odd integer, and $D$ is a positive integer corresponding to the amount of delay reduction. From (16), the estimated value of $L_{Q}$ is increased until the required specification is met, since slightly longer subfilter length is usually required as compared to its linear-phase counterpart for the same specification. With a given subfilter length, the design problem is to approximate the desired response $Q_{d}(\omega)$ by $Q\left(e^{j \omega}\right)$ in the minimax sense by minimizing:

$$
E_{\infty}=\max _{\omega}\left|Q\left(e^{j \omega}\right)-Q_{d}(\omega)\right|, \omega \in\left[0,2 \omega_{c}\right] .
$$

The minimization problem can be solved readily using SOCP [9]. Due to page limitation, interested readers are referred to [13] for more details.

\section{DESIGN EXAMPLES}

\section{Example 1: PR Linear-Phase Multi-plet FBs}

In this example, two-channel linear-phase FIR multi-plet FB with the following specifications is designed using the proposed approach: cutoff frequency $\omega_{c}=0.4 \pi$, passband ripples $\delta_{p 0}=\delta_{p 1}$ $=3.45394 \times 10^{-5}$ (i.e. $3 \times 10^{-4} \mathrm{~dB}$ passband deviation) and stopband ripples $\delta_{s 0}=\delta_{s 1}=0.00316$ (i.e. $-50 \mathrm{~dB}$ stopband attenuation). For these specifications, a low order prototype FB with $L=4$ lifting steps is first designed and the corresponding frequency response is shown in figure 3a. The corresponding lifting coefficients and scaling constants are listed in the second column of table 1 . From figure $3 \mathrm{a}$, it can be seen that $\widetilde{\omega}_{c}$ should be chosen as $0.04 \pi$ so as to satisfy the prescribed passband and stopband ripples. In order for the subfilter to satisfy (12), its filter length is chosen to be 16 according to (16). The frequency response of the transformed FB is shown in figure $3 \mathrm{~b}$. The specifications of the subfilter and the design results in this example are summarized in tables 2 and 3, respectively. To illustrate the flexibility of the proposed transformation method, figures $3 \mathrm{c}$ and $3 \mathrm{~d}$ show the frequency responses of the subfilters with different cutoff frequencies and the corresponding transformed multi-plet FBs. It can be seen that the passband and stopband are equiripple and the frequency response is very symmetry. Furthermore, by realizing the subfilter as variable digital filters [8], the cutoff frequencies can be varied online. For comparison purpose, we also consider the design of the triplet FB proposed in [5]. The general design procedure of the triplet FB requires three separate designs of the subfilters and their lengths have to be chosen properly so as to satisfy different specifications. By a number of trials and errors, we found that the lengths of these three subfilters have to be 22 so as to satisfy the same specifications above with the minimum arithmetic complexity. A similar result can also be obtained by designing a single subfilter of length 22 using the proposed approach and the prototype FB in the triple structure as shown in table 1. The design results are omitted due to page limitation. This triplet FB has a comparable arithmetic complexity and performance as the aforementioned multi-plet FB. On the other hand, since there is no systematic design procedure for determining the subfilter lengths, one would have to try every combination of the subfilter lengths in order to meet the specification. This is rather time consuming and makes the design inefficient. For the proposed approach, the frequency response of the prototype PR FB, such as the passband and stopband ripples, can be controlled through optimization with few variables. Identical subfilters can then be designed separately to meet the specification.

\section{Example 2: PR Low-Delay Multi-plet FBs}

This example illustrates the flexibility of the proposed approach in designing low-delay multi-plet PR FB. The specifications are: $5 \times 10^{-4} \mathrm{~dB}$ passband deviation, $-50 \mathrm{~dB}$ stopband attenuation, $\omega_{c}=0.45 \pi$ and $D=10$. From (5), the group delays of the desired analysis lowpass and highpass filters are significantly reduced to 63 and 84 samples, as compared to 93 and 124 samples for the linear-phase case with $D=0$. It was found that a subfilter with $\widetilde{\omega}_{c}=0.04 \pi$ and $L_{Q}=32$ is able to meet the required specifications. The third column of table 2 summarizes the parameters of the subfilter. The frequency and group delay responses of the transformed multi-plet FB are respectively shown in figures $4 \mathrm{a}$ and $4 \mathrm{~b}$. It can be seen that the low-delay multi-plet FB is approximately linear-phase in the passband with peak group delay errors of 0.0083 samples for $H_{0}(z)$, and 0.0086 samples for $H_{1}(z)$. Figures $4 \mathrm{c}$ and $4 \mathrm{~d}$ show the analysis scaling and wavelet functions. They are rather smooth, but not symmetric due to the low-delay constraint. The performances of the transformed FB are summarized in table 3 .

\section{CONCLUSION}

A new class of two-channel structural PR FIR FBs called multi-plet FBs is presented. It generalizes structural PR FBs and triplet FBs by employing multiple lifting steps in the conventional lifting structure. The design of the multi-plet FBs can be done in two separate steps: (i) a low order prototype PR FB with much wider transition band is first designed to satisfy prescribed passband/stopband ripples, and (ii) the prototype FB is then wrapped by means of frequency transformation through an appropriate subfilter to meet the desired transition bandwidth, while preserving the PR condition, passband/stopband ripples and lifting structure.. The use of low-delay subfilters is also proposed to further reduce the system delay of the multi-plet FBs

\section{REFERENCES}

[1] S. M. Phoong, C. W. Kim, P.P. Vaidyanathan and R. Ansari, “A new class of two-channel biothogonal filter banks and wavelet bases," IEEE Trans. Signal Processing, vol. 43, pp. 649-664, 1995.

[2] R. Ansari, C. W. Kim and M. Dedovic, "Structure and design of two-channel filter banks derived from a triplet of halfband filters," IEEE Trans. Circuits Syst. II, vol. 46, no. 12, pp. 1487-1496, 1999. 
[3] I. Daubechies and W. Sweldens, "Factoring wavelet transform into lifting steps," J. Fourier Anal. Appl., vol. 4, no. 3, pp. 247-269, 1998.

[4] D. B. H. Tay, "Least squares design of the class of triplet halfband filter banks," in Proc. ISCAS'2001, vol. 2, pp. 481-484, 2001.

[5] K. S. Yeung and S. C. Chan, "On the Design and Multiplier-less Realization of Perfect Reconstruction Triplet-based FIR Filter Banks and Wavelet Bases," IEEE Trans. Circuits Syst. I, vol. 51, pp. 1476-1491, Aug. 2004.

[6] T. Saramaki, "Design of FIR filters as a tapped cascaded interconnection of identical subfilters," IEEE Trans. Circuits Syst., vol. 34, pp. 1011-1029, Sep. 1987.

[7] J. F. Kaiser and R. W. Hamming, "Sharpening the response of a symmetric nonrecursive filter by multiple use of the same filter," IEEE Trans. Acoust. Speech, Signal Processing, vol. 25, pp. 415422, Oct. 1977.

[8] K. S. Pun, S. C. Chan, K. S. Yeung and K. L. Ho, "On the Design and Implementation of FIR and IIR Digital Filters with Variable Frequency Characteristics," IEEE Trans. Circuits Syst. II, vol. 49, no. 11, pp. 689-703, Nov, 2002.

[9] S. C. Chan, C. K. S. Pun and K. L. Ho, "New Design and Realization Techniques for a Class of Perfect Reconstruction TwoChannel FIR Filterbanks and Wavelets Bases," IEEE Trans. Signal Processing, vol. 52, pp. 2135 - 2141, July 2004.

[10] M. S. Lobo, L. Vandenberghe, S. Boye and H. Lebret, "Applications of second-order cone programming," Linear Algebra Application, vol. 248, pp. 193-228, Nov. 1998.

[11] D. B. H. Tay, "Design of perfect reconstruction filter banks using transformation of variables and re-factorization," in Proc. ICICS'1997, vol. 3, pp. 1349-1352, Sept. 1997.

[12] T. G. Marshall, Jr., "Zero-phase filter bank and wavelet coder matrices: properties, triangular decompositions, and a fast algorithm" Multidimensional Syst. Signal Processing: Special Issue on Wavelets and Multiresolution Signal Processing, vol. 7, 1996.

[13] S. C. Chan and K. M. Tsui, "Multi-plet two-channel perfect reconstruction filter banks," internal report, Department of Electrical and Electronic Engineering, the University of Hong Kong.

\begin{tabular}{||c|c|c|}
\hline & Prototype FB & Triplet FB [4,5] \\
\hline$p_{0}$ & 0.23987556667257 & $1-\sqrt{2}$ \\
\hline$p_{1}$ & -0.54571527976115 & $1 / \sqrt{2}$ \\
\hline$p_{2}$ & 0.54045911345798 & $1-\sqrt{2}$ \\
\hline$p_{3}$ & -0.23167459250035 & N/A \\
\hline$C_{0}$ & 0.71237102180672 & $1 / \sqrt{2}$ \\
\hline$C_{1}$ & 0.71401331291255 & $1 / \sqrt{2}$ \\
\hline
\end{tabular}

Table 1. Lifting coefficients and scaling constants of the prototype FBs.

\begin{tabular}{||c|c|c||}
\hline & Ex. 1 & Ex. 2 \\
\hline$L_{Q}$ & 16 & 32 \\
\hline$D$ & 0 & 10 \\
\hline$\omega_{c}$ & $0.4 \pi$ & $0.45 \pi$ \\
\hline$\widetilde{\omega}_{c}$ & $0.04 \pi$ & $0.04 \pi$ \\
\hline
\end{tabular}

Table 2. Specifications of the subfilters in examples 1 and 2.

\begin{tabular}{||l|c|c||}
\hline & Ex. 1 & Ex. 2 \\
\hline Group Delays of $H_{0}(z), H_{1}(z)$ & 45,60 & 63,84 \\
\hline Passband deviation of $H_{0}(z) / 10^{-3} \mathrm{~dB}$ & 0.2950 & 0.3503 \\
\hline Stopband attenuation of $H_{0}(z) / \mathrm{dB}$ & 53.0565 & 51.3018 \\
\hline Group delay error of $H_{0}(z) / \mathrm{samples}$ & 0 & 0.0083 \\
\hline Passband deviation of $H_{1}(z) / 10^{-3} \mathrm{~dB}$ & 0.2965 & 0.3530 \\
\hline Stopband attenuation of $H_{1}(z) / \mathrm{dB}$ & 53.0219 & 51.4734 \\
\hline Group delay error of $H_{1}(z) / \mathrm{samples}$ & 0 & 0.0086 \\
\hline
\end{tabular}

Table 3. Design results of the transformed FBs in examples 1 and 2.
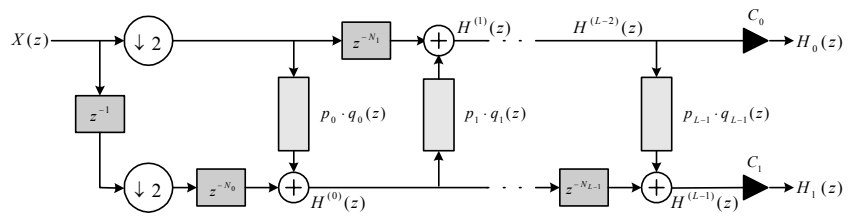

1a)

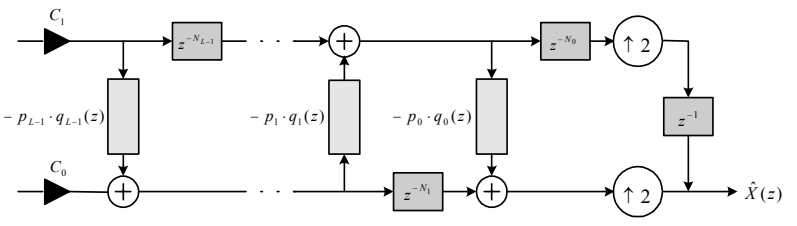

1b)

Figure 1: Structure of the multi-plet FBs: (a) Analysis bank. (b) Synthesis bank.

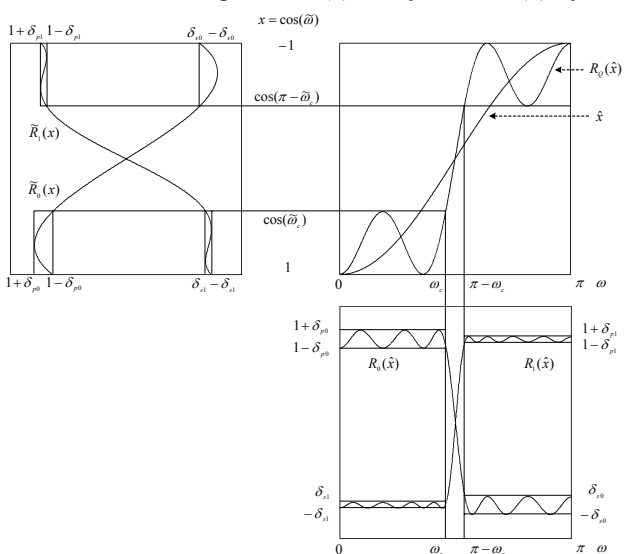

Figure 2: General relations and specifications of the prototype FB, the subfilter and the multi-plet transformed FB.

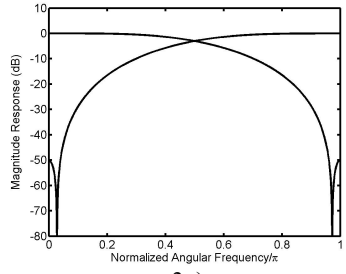

3a)

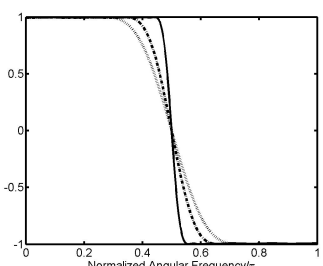

$3 c)$

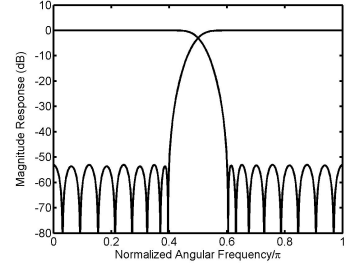

3b)

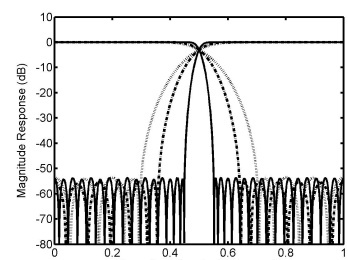

$3 \mathrm{~d})$
Figure 3. Design results of FIR linear-phase multi-plet FB in example 1: a) Frequency response of the prototype FB. b) Frequency response of the transformed FBs with $\omega_{c}=0.4 \pi$. c) and d) Magnitude responses of the subfilters and the corresponding frequency response of the transformed FBs with different cutoff frequencies (dotted line: $\omega_{c}=0.3 \pi$, dash-dotted line: $\omega_{c}=0.36 \pi$ and solid line: $\left.\omega_{c}=0.45 \pi\right)$.

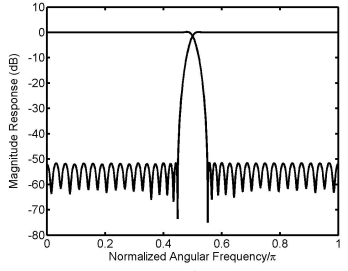

4a)

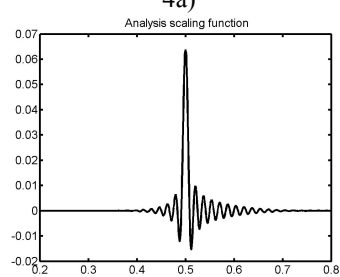

$4 c)$

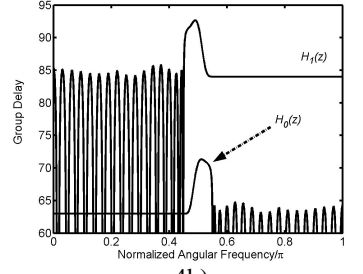

$4 \mathrm{~b})$

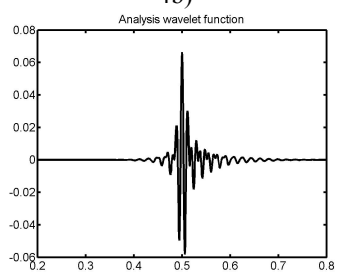

4d)
Figure 4. Design results of FIR low-delay multi-plet FB in example 2: a) Frequency responses, b) group delay response, c) analysis scaling function and d) analysis wavelet function of the transformed FB with $\omega_{c}=0.45 \pi$ and $D=10$. 